\title{
Glossário para a colaboração intersetorial: Conceitos selecionados
}

\author{
Intersectoral collaboration glossary: Selected concepts \\ Glosario de colaboración intersectorial: Conceptos seleccionados
}

Recebido: 09/07/2021 | Revisado: 15/07/2021 | Aceito: 17/07/2021 | Publicado: 26/07/2021

\author{
Antônio Paulo Gomes Chiari \\ ORCID: https://orcid.org/0000-0002-4104-9164 \\ Universidade Federal de Minas Gerais, Brasil \\ E-mail: apgchiari@gmail.com \\ Viviane Elisângela Gomes \\ ORCID: https://orcid.org/0000-0001-9637-1911 \\ Universidade Federal de Minas Gerais, Brasil \\ E-mail: vivigomes_br@yahoo.com.br \\ Anna Rachel dos Santos Soares \\ ORCID: https://orcid.org/0000-0002-1232-1712 \\ Universidade Federal de Minas Gerais, Brasil \\ E-mail: anna.soares@outlook.com \\ Geraldo Cunha Cury \\ ORCID: https://orcid.org/0000-0002-0411-8343 \\ Universidade Federal de Minas Gerais, Brasil \\ E-mail: geraldocunhacury@gmail.com \\ Maria Inês Barreiros Senna \\ ORCID: https://orcid.org/0000-0002-0578-8744 \\ Universidade Federal de Minas Gerais, Brasil \\ E-mail: mariainessenna@gmail.com \\ Raquel Conceição Ferreira \\ ORCID: https://orcid.org/0000-0001-8897-9345 \\ Universidade Federal de Minas Gerais, Brasil \\ E-mail: raquelconceicaoferreira@gmail.com
}

\begin{abstract}
Resumo
Este estudo teórico reconstrói as principais categorias conceituais que orientam a ação intersetorial, a partir da experiência empírica dos autores cotejada com a literatura sobre o tema. O texto está organizado como um glossário e apresenta treze termos selecionados: agenda, colaboração intersetorial, conflito, governança, governança colaborativa, indivíduo, mecanismo integrador, modelo de gestão, parceria, processo comunicativo, setor, sustentabilidade, território. A partir desses conceitos estruturantes foi desenvolvido um modelo das dimensões da colaboração intersetorial: dimensão política, dimensão técnica e dimensão da interação. Todas elas inter-relacionadas e produtoras de mecanismos de integração e de sustentação para a colaboração intersetorial. Espera-se oferecer repertório e campos de possibilidades que, ao promoverem um melhor entendimento da prática intersetorial, facilitarão o uso desta estratégia no desenvolvimento de políticas públicas de corte social, assim como contribuirão no delineamento de pesquisas sobre o tema. As definições e reflexões daqui extraídas confirmam a complexidade da colaboração intersetorial e a necessidade da busca de suporte em diferentes áreas do conhecimento, tanto para sua construção quanto para sua efetivação como prática geradora de equidade e justiça social.
\end{abstract}

Palavras-chave: Colaboração intersetorial; Política pública; Promoção da saúde; Inovação.

\begin{abstract}
This article analyzes the main conceptual categories that guide intersectoral action based on the authors empirical experience compared to the literature on the subject. The text is organized as a glossary and presents thirteen terms: agenda, intersectoral collaboration, conflict, governance, collaborative governance, individual, support mechanism, management model, partnership, communication process, sector, sustainability, territory. These concepts enabled the development of a model of the dimensions of intersectoral collaboration: political dimension, technical dimension, and interaction dimension. They are interrelated and produce integration and support mechanisms for intersectoral collaboration. This theoretical contribution aims to promote a better understanding of intersectoral practice facilitating the choice of this approach in the development of public social policies. Additionally, the covered concepts may contribute to the design of research in the area. The definitions and reflections confirm the complexity of intersectoral collaboration and the importance of seeking support in different knowledge fields for its construction and effectiveness as a practice that generates equity and social justice.
\end{abstract}

Keywords: Intersectoral collaboration; Public policy; Health promotion; Innovation. 


\begin{abstract}
Resumen
Este artículo analiza las principales categorías conceptuales que orientan la acción intersectorial, a partir de la experiencia empírica de los autores en comparación con la literatura sobre el tema. El texto está organizado como un glosario y presenta trece términos: agenda, colaboración intersectorial, conflicto, gobernanza, gobernanza colaborativa, individuo, mecanismo integrador, modelo de gestión, alianza, proceso de comunicación, sector, sostenibilidad, territorio. A partir de estos conceptos estructurantes, se desarrolló un modelo de las dimensiones de la colaboración intersectorial: dimensión política, dimensión técnica y dimensión de interacción. Todos ellos están interrelacionados y producen mecanismos de integración y apoyo a la colaboración intersectorial. Promoviendo un mejor entendimiento de la práctica intersectorial, este aporte teórico tiene como objetivo ofrecer repertorio y posibilidades que faciliten el uso de esta estrategia en el desarrollo de políticas públicas sociales, así como contribuir al diseño de investigaciones en el área. Las definiciones y reflexiones confirman la complejidad de la colaboración intersectorial y la importancia de buscar apoyos en diferentes campos del conocimiento, para su construcción y efectividad como práctica generadora de equidad y justicia social.
\end{abstract}

Palabras clave: Colaboratión intersectorial; Política pública; Promotión de la salud; Innovación.

\title{
1. Introdução
}

A colaboração intersetorial é recomendada por várias políticas e programas de governo, e tem sido objeto de investigações acadêmicas em vários países (Dubois, et al., 2015; Glandon, et al., 2019; Shankardass, et al., 2012). Atualmente, é descrita como estratégia fundamental para implementação de políticas sociais, especialmente para a promoção da saúde e para o combate às iniquidades (Freiler, et al., 2013; McDaid \& Park, 2016; Solar, et al., 2009). Indo além, o décimo sétimo objetivo de Desenvolvimento Sustentável da Agenda 2030 da Organização das Nações Unidas propõe o fortalecimento dos meios de implementação e revitalização das parcerias globais para o desenvolvimento sustentável (United Nations, 2019).

Apesar de todo o conhecimento produzido no campo científico e no campo das práticas, superar o "dilema da intersetorialidade" não tem sido uma tarefa fácil (Andrade, 2006). A primeira definição de colaboração intersetorial ocorreu no campo da economia (Dubois, et al., 2015). Solar, et al. (2009), propuseram a construção de uma tipologia para a colaboração intersetorial no combate às iniquidades em saúde. Esta construção subsidiaria um melhor delineamento das práticas intersetoriais. Akerman, et al. (2014), discorreram sobre a polissemia do termo e sobre a existência de um "ativismo intersetorial" ainda frágil e pouco consistente. Propuseram uma série de caminhos, compostos por perguntas de pesquisa, que contribuiriam para a análise e para a adoção de práticas intersetoriais integradas. O próprio conceito de colaboração intersetorial permanece obscuro e contestado, merecendo análise crítica mais aprofundada. Da mesma forma, sua prática cotidiana nas políticas públicas, seus sucessos e fracassos, permanecem em grande parte sem exame (Chircop, et al., 2015; Dubois, et al., 2015). Neste mesmo sentido, Holt, et al. (2018), avaliaram que as recomendações encontradas na literatura oferecem uma conceituação muito abstrata sobre a ação intersetorial e, consequentemente, fornecem poucas diretrizes práticas para a ação.

As lacunas em torno do conceito de colaboração intersetorial e as dificuldades de atuação a elas associadas foram os estímulos para a elaboração deste texto. Como buscar uma práxis sobre a qual pouco se sabe? Sem ter a proposta de esgotar o tema, o glossário traz alguns dos termos mais relevantes para a compreensão teórica e prática da colaboração intersetorial. Ao escolher e trabalhar teoricamente estes termos, o objetivo foi fornecer repertório e referências, ampliando a compreensão e o campo de possibilidades para a construção intersetorial. Traçando estas referências e trazendo-as para o debate, buscou-se contribuir para o fortalecimento dos operadores das ações intersetoriais - gestores e trabalhadores. Para os pesquisadores, principalmente aqueles iniciantes no estudo deste tema, fica a oferta de um ordenamento conceitual que poderá auxiliar no delineamento de novos objetos de pesquisas, assim como na análise das experiências em curso.

Respeitando a ordenação de um glossário, os termos estão dispostos em ordem alfabética. Esta opção, que a princípio parece ter sacrificado o desenvolvimento lógico do tema, manteve coerência com a proposta inicial de ser um instrumento de consulta teórico-metodológico para a prática intersetorial. Ao final, à guisa de sistematização, desenvolvemos um modelo das 
dimensões da colaboração intersetorial visando contribuir para a proposição, implementação e avaliação de políticas, ações e pesquisas.

\section{Breve Percurso Metodológico}

Estudo teórico que surgiu da intersecção dos vários caminhos traçados em um processo de pesquisa. Buscava-se aprofundar o estudo e as reflexões sobre a colaboração intersetorial e, simultaneamente, analisar as interações entre os vários atores envolvidos na promoção do desenvolvimento infantil, tema ao qual a colaboração intersetorial está relacionada na pesquisa de campo ora realizada. Experiências de estudos de casos anteriores, envolvendo implementação e operacionalização de políticas, projetos e ações intersetoriais, também se encontram refletidas nos termos abordados neste texto. Buscou-se formar uma correlação entre a construção pessoal dos autores e os vários pontos de vista distintos encontrados na literatura, num processo hermenêutico-dialético de reconstituição de construções (Guba \& Lincoln, 2011).

Um glossário pareceu, então, uma ferramenta pertinente e inovadora para a sistematização das principais categorias conceituais que orientam a ação intersetorial; um instrumento de revisão e de reflexão sobre suas dimensões, seus componentes essenciais, assim como fatores e contextos determinantes. Experiências intersetoriais identificadas durante a revisão não sistemática da literatura foram citadas para ilustrar algumas das definições abordadas.

\section{Glossário}

\section{Agenda}

Aqui se apresentam duas questões: a agenda como pauta definida previamente e a agenda como reserva de tempo cronológico. Estes dois aspectos refletem a importância e a necessidade de espaços e instâncias onde os vários setores possam expressar seus interesses, diferenças e conflitos (Cunill Grau, 2014). Entendimento e valorização institucionais da proposta de trabalho intersetorial são fatores definidores da agenda de trabalho sobre o tema.

A definição e manutenção da pauta de discussão se relacionam com a dimensão política da colaboração intersetorial; é o status de sua prioridade na agenda institucional. A agenda cronológica se refere ao tempo necessário para o processo de construção da colaboração intersetorial: encontros, conversas, planejamento, avaliação. A efetivação de uma agenda comum a todos os setores envolvidos será tão mais fácil, quanto maior for o apoio político à proposta. A definição de uma agenda para o processo de construção de uma colaboração intersetorial não é simples, nem mesmo no âmbito de políticas intersetoriais institucionais (Quadro 1). 
Quadro 1 - Agenda, conflito e mecanismo integrador a partir da colaboração intersetorial no Programa Saúde na Escola.

O Programa Saúde da Escola (PSE) foi instituído no Brasil em 2007 e tem como objetivo a formação integral dos alunos da rede pública de ensino. O programa tem como fundamento o trabalho articulado entre educação, saúde e outros setores e tem sido objeto de estudos para a compreensão dos mecanismos da colaboração intersetorial (Brasil, et al., 2017; Chiari, et al., 2018; Ferreira, et al., 2014; Sousa, et al., 2017).

Agenda: Nos quatro estudos analisados, gestores e trabalhadores queixaram-se da dificuldade de compatibilizar as agendas para as ações do programa. As agendas de cada setor eram muito intensas e direcionadas para suas atividades específicas (Brasil, et al., 2017; Chiari, et al., 2018; Ferreira, et al., 2014; Sousa, et al., 2017). Para o grupo de gestores do PSE em uma capital brasileira, mesmo havendo reconhecimento e valorização institucionais do programa, a agenda intersetorial não teve relevância política suficiente para possibilitar a construção de compartilhamento de poderes (Chiari, et al.,2018).

Conflito: A explicitação de conflitos e divergências apareceu em três dos quatro estudos analisados (Brasil, et al., 2017; Chiari, et al., 2018; Ferreira, et al., 2014). Em dois deles, foram identificadas controvérsias em relação à tomada de decisões na gestão do PSE, assim como abuso de protagonismo de determinado setor sobre os outros (Chiari, et al.,2018; Ferreira, et al., 2014). Enquanto o segundo trabalho avaliou que o grupo de gestores estava passando por um processo de aprendizagem para o trabalho intersetorial, o primeiro identificou que os membros da gestão municipal do PSE não conseguiram utilizar o grupo de trabalho intersetorial como espaço de negociação em prol da superação das diferenças.

Mecanismo integrador: Foram identificados mecanismos integradores facilitadores da construção intersetorial dentro do PSE, seja no arcabouço normativo do programa, seja nas propostas de organizações locais. Gestão colegiada do programa, compartilhamento de responsabilidades, trabalho conjunto com troca de saberes e de experiências, capacitações e articulações conjuntas no território - para apropriação de linguagens, de nivelamento técnico e conceitual, de fomento à discussão, de qualificar a comunicação - são alguns exemplos citados pelos autores. As experiências analisadas mostraram, no entanto, pouca utilização destes mecanismos identificados (Brasil, et al., 2017; Chiari, et al., 2018; Ferreira, et al., 2014; Sousa, et al., 2017). O desconhecimento de importantes componentes do programa foi uma queixa explícita dos trabalhadores entrevistados por Brasil, et al. (2017), e percebido nas respostas dos gestores municipais do PSE ao questionário aplicado por Chiari, et al. (2018).

Fonte: Autores.

\section{Colaboração intersetorial}

Colaboração é entendida como a forma mais desenvolvida de comunicação interativa, exigindo que os atores tenham estratégia, identidade, objetivos e responsabilidades conjuntas. Definições encontradas na literatura descrevem no conceito de colaboração a organização de atores em rede, seus objetivos compartilhados e suas negociações contínuas para harmonizar interesses comuns e coordenar atividades mútuas (Russell \& Smorodinskaya, 2018). Neste sentido a colaboração intersetorial pode ser definida como estratégia de gestão voltada para a articulação de saberes, potencialidades e experiências de indivíduos, grupos e setores (governamentais ou não), visando ao enfrentamento de problemas sociais complexos, por meio de intervenções compartilhadas e com efeitos sinérgicos. Pressupõe formação de vínculos, corresponsabilidades e cogestão na busca de objetivos comuns orientados ao interesse público (Brasil, 2014; Cunill Grau, 2005; Inojosa, 2001; Monnerat \& Souza, 2014).

Intersetorialidade tem sido o termo mais utilizado na literatura científica brasileira. Prevalece também nos textos das políticas públicas e entre os profissionais nos serviços. Apesar de menos comum, alguns textos em língua inglesa dos anos 2000 também utilizavam o termo correspondente (intersectorality) (Solar, et al., 2010). Mais recentemente, Dubois, et al. (2015), identificaram dez diferentes termos para se referir ao tema. Optaram por quatro deles como critério de elegibilidade para a revisão realizada: ação intersetorial, ação intersetorial para a saúde, colaboração intersetorial, política intersetorial. Atualmente, na literatura em língua inglesa o termo mais comum é o correspondente à colaboração intersetorial (intersectoral collaboration). Percebe-se no Brasil uma tendência neste mesmo sentido, embasada provavelmente na indexação deste termo junto aos Descritores em Ciências da Saúde da Biblioteca Virtual em Saúde (BVS, DeCS). Treze outros termos são reconhecidos como sinônimos, em português, inclusive intersetorialidade.

Cabe, ainda, ressaltar que a colaboração intersetorial não é uma estratégia restrita e exclusiva para enfrentamento dos problemas de saúde. Deve ser entendida como uma possibilidade de atuação em todas as políticas públicas sempre que houver 
a necessidade de planejamento participativo e integrado para a solução sinérgica de problemas complexos (Bronzo, 2016; Monnerat \& Souza, 2011).

\section{Conflito}

No processo da interação entre os setores, dissensos e tensões devem ser usados produtivamente na busca por soluções integradas para resolver problemas sociais complexos. Este é o fundamento técnico da colaboração intersetorial (Cunill Grau, 2014). O conflito não é negativo. Sendo uma interação entre indivíduos, o conflito é uma síntese de elementos no caminho para a resolução das tensões entre as partes contrárias. A rejeição e a indiferença é que devem ser combatidas, pois estas sim representam a supressão da interação e a falta de interesse pela busca de um objetivo comum (Simmel, 1995). Tensões latentes, não articuladas e, consequentemente não trabalhadas, tendem a produzir responsabilidades difusas e nenhuma prioridade na formulação de políticas intersetoriais (Holt, et al., 2018). Além disso, iniciativas colaborativas que são incapazes de abordar os conflitos e deliberar sobre eles, podem simplesmente produzir listas de intenções ou simples acordos com declarações vagas e evasivas, em grande parte ocultando as contradições fundamentais (Bodin, 2017).

A superação do conflito pela via da argumentação possibilita a mobilização de poderes e forças. As controvérsias não devem ser encaradas como um revés a ser evitado. O seu enfrentamento, análise e resolução possibilitarão o estabelecimento de uma via de interação social com reconfiguração da rede (Latour, 2012; Rivera \& Artmann, 2016). Esse processo contribui no desenvolvimento de soluções novas e mais duráveis que possibilitam melhor colaboração (Quadro 1).

\section{Governança}

Governança pode ser definida como a capacidade do Estado de implementar as políticas públicas necessárias para se alcançar os objetivos comuns. Compreende a interlocução com a sociedade por meio de ações que garantam a participação dos cidadãos e a responsabilização dos agentes públicos (Oliveira \& Pisa, 2015). A governança pressupõe a identificação dos problemas da sociedade, a formulação de políticas que os solucione e a implementação das ações propostas, pela mobilização dos meios e recursos necessários (Santos, 1997).

A prática intersetorial necessita da criação de formas de trabalho que adotem estruturas e regras de governança de rede. Um modelo de governança inovador pode estimular novas lógicas e arranjos promotores de experiências exitosas (Martins, et al., 2019). Deve haver o estabelecimento de vínculos entre os indivíduos envolvidos e a organização do trabalho coletivo. Um pressuposto fundamental da governança é a responsabilização com transparência em todas as etapas do processo, entre os atores envolvidos nas ações, assim como destes com os indivíduos alvos dos projetos desenvolvidos (Quadro 2). 
Quadro 2 - Governança, governança colaborativa, indivíduo, parceria e território: a experiência canadense com os Comitês de Vizinhanças e os Mapas para planejamento e ação intersetorial

Estudos desenvolvidos no Canadá demonstraram que intervenções sobre os determinantes sociais da saúde, por meio da ação intersetorial local, podem levar a transformações concretas nos ambientes vivos. Bilodeau, et al., (2019) conduziram um estudo de caso com os Comitês de Vizinhanças na cidade de Montreal, com o objetivo de examinar como os efeitos da ação intersetorial são produzidos e podem ser atribuídos aos processos interativos. O Conselho Canadense de Determinantes Sociais da Saúde (CCSDH, sigla em inglês) produziu um relatório que apresentou dois estudos de caso de iniciativas de mapeamento colaborativo que integraram dados sociais e de saúde. Os relatos enfocaram as maneiras como as ferramentas de mapeamento e compartilhamento de dados contribuíram para decisões baseadas em evidências para a ação intersetorial (CCSDH, 2014).

Governança: A organização do trabalho coletivo possibilitou a adoção de estruturas e regras de governança de rede. Elas dizem respeito às ferramentas e práticas que regularam a participação das partes envolvidas, a legitimidade de seus porta-vozes e o processo coletivo de tomada de decisões (Bilodeau, et al., 2019).

Governança colaborativa: Clientes, parceiros e colaboradores contribuíram com o projeto participando de comitês de governança e apoiando de maneira concreta as atividades. Essa abordagem centrada no usuário permitiu aos envolvidos expressar suas necessidades e encontrar as respostas adequadas às mesmas. Todos investiram tempo e esforço para aumentar a conscientização sobre a iniciativa e promover relacionamentos (CCSDH, 2014).

Indivíduo: Foi identificada a importância da movimentação e do compromisso dos indivíduos (atores, segundo termo utilizado pelas autoras) na produção dos resultados da ação intersetorial. Os movimentos surgiram das negociações e dos jogos de poder entre eles e envolveram mudanças de posição (opiniões, pontos de vista), atuação em novos papéis e transformações nas relações de poder. A transformação eficaz dependeu ainda do compromisso daqueles que detinham as rédeas da tomada de decisão e da ação, dentro ou fora de uma rede (por exemplo, serviços municipais, empresas, organizações sem fins lucrativos, organizações comunitárias) (Bilodeau, et al., 2019).

Parceria: Os projetos de mapeamento trabalharam com os determinantes sociais da saúde, mas nenhum deles enquadrou o trabalho exclusivamente em termos de questões de saúde. Pelo contrário, os dados estavam disponíveis em uma ampla gama de tópicos. Definir a iniciativa de forma mais ampla, em vez de focar exclusivamente na saúde, ajudou a construir apoio e engajar parceiros. As parcerias contribuíram para a geração de recursos, credibilidade e sustentabilidade. As experiências indicaram a necessidade e importância de esforços dedicados à tarefa de manter os parceiros informados e engajados (CCSDH, 2014).

Território: A agregação de dados das áreas geográficas específicas garantiu que eles se encaixassem nas estruturas de tomada de decisão pré-existentes. O contexto institucional e territorial no qual a iniciativa esteve inserida foi levado em consideração. A ênfase colocada nesses limites territoriais produziu sentido para os usuários e foram compatíveis com as necessidades locais (Bilodeau, et al., 2019; CCSDH, 2014).

Fonte: Autores.

\section{Governança colaborativa}

A governança colaborativa envolve a colaboração de diferentes organizações dos setores públicos, privados e da sociedade civil para o alcance de objetivos compartilhados. O consenso deliberativo e as decisões coletivas constituem a base da governança colaborativa (Ran \& Qi, 2018) e são arranjos determinantes da abordagem intersetorial na formulação e na implementação de ações públicas. A governança colaborativa contribui na solução de problemas públicos complexos na medida em que integra e amplia recursos, promove troca de informações e compartilhamento de conhecimento, motiva a inovação, promove formas mais efetivas, eficientes, flexíveis e sofisticadas de solucionar problemas. Como resultado, pode gerar soluções que capturam os interesses e as necessidades das diferentes partes interessadas.

Assim, a eficácia de uma rede de governança colaborativa resulta da interação entre a estrutura geral da rede, as características de seus atores e as posições na rede que eles ocupam. Consequentemente, a governança por meio da colaboração de múltiplos atores/setores é, em comparação com os modos mais tradicionais e burocráticos de governo, imbuída de questões críticas relativas a várias qualidades democráticas, como transparência dos procedimentos de tomada de decisão, legitimidade, responsabilidade e justiça (Bodin, 2017) (Quadro 2).

\section{Indivíduo}

As ciências sociais apresentam uma série de termos para designar o ser humano em seus processos interativos na sociedade. Tomaremos emprestado da sociologia, mais especificamente de Martuccelli (2002), o termo indivíduo, na acepção 
de membro singular de uma coletividade. Segundo o autor, o indivíduo ocupa posição central no mundo contemporâneo e sua atuação na sociedade é guiada por algumas contradições: é um ser único e independente, mas inseparável do entorno e dos outros indivíduos; é portador de autonomia e liberdade, mas ao mesmo tempo com condutas delimitadas pelo contexto social; age segundo sua subjetividade, mas também condicionado por uma força histórica e normativa (Martuccelli, 2002).

Estes aspectos do comportamento do indivíduo em sociedade precisam ser levados em conta nos processos de implementação de políticas públicas. São eles os idealizadores, promotores, executores e beneficiários das políticas. São as partes interessadas - stakeholders na literatura em inglês - e este interesse possui nuances relacionadas às contradições e ambiguidades estruturantes dos indivíduos. Neste sentido, deve-se ficar atento à posição ocupada por cada indivíduo no processo de implementação de um projeto ou de uma política (intersetorial ou não): quem são eles, com quem eles colaboram e como as estruturas das redes de colaboração intersetorial se relacionam com as habilidades dos indivíduos para lidar com diferentes problemas. Governantes, gestores, trabalhadores, beneficiários atuarão com visões, acessos e instrumentos diferenciados e toda essa diversidade deverá ser equacionada de alguma forma ao longo da articulação (Bodin, 2017; Monnerat \& Souza, 2011). Esta ação articulada será facilitada pela presença de lideranças que tenham visão ampliada e atuação em vários níveis. É necessário um conjunto específico de habilidades que envolvem capacidade para resolução de problemas, coordenação e intermediação, além de flexibilidade, conhecimento profundo do sistema e disposição para realizar o trabalho emocional associado ao trabalho relacional (Holt, et al., 2018). A experiência canadense evidencia a importância da movimentação e dos compromissos dos indivíduos no processo de colaboração intersetorial (Quadro 2).

\section{Mecanismo integrador}

Mecanismos integradores constituem um arsenal de práticas, estratégias, ferramentas ou dispositivos potencialmente promotores da ação coletiva necessária à realização de políticas e programas governamentais. São instrumentos que sustentam e organizam as relações no cotidiano da implementação de uma ação intersetorial. Eles contribuem na superação do isolamento e da desarticulação dos setores e podem estimular visão e construção solidárias ao propiciar gestão integrada e formas matriciais de organização. Ao viabilizarem a conversa entre os setores envolvidos, assim como a participação dos cidadãos, são capazes de minimizar as diferenças percebidas de poder e contribuir para o estabelecimento de comunidades de significados (Cunill Grau, 2005; Pires, 2016). As próprias etapas de implementação de uma política intersetorial podem ser entendidas como mecanismos de integração, na medida em que houver um processo coletivo e compartilhado de definição de problemas, planejamento de ações, definição de indicadores e avaliação com foco nos resultados (Rasanathan et al., 2017). Estudos recentes têm demonstrado o papel relevante da liderança colaborativa e compartilhada na condução de ações intersetoriais para a promoção da saúde (Holt, et al., 2018; Kuruvilla, et al., 2018; Rasanathan, et al., 2017).

Processos de educação permanente são um mecanismo integrador privilegiado por viabilizar a aproximação entre os gestores e trabalhadores dos setores envolvidos. Operam como locus para ambientação em relação às linguagens e às formas de atuação, próprias de cada setor. Podem e devem ser aproveitadas, inclusive, para discussão conceitual sobre a colaboração intersetorial. É recomendável que capacitações e treinamentos estejam inseridos no delineamento dos projetos e das políticas intersetoriais e estes devem ser contextualizados a partir dos problemas que se pretende enfrentar. Ao mesmo tempo em que são um desafio para gestores e tomadores de decisões, pois são processos que demandam recursos de várias ordens, são um campo fértil para as interações que poderão resultar em parcerias efetivamente intersetoriais (Chiari, et al., 2021).

Muitas vezes os mecanismos integradores estão previstos na concepção do projeto ou da política, mas não são efetivamente utilizados, ou são esquecidos nas rotinas fragmentadas do trabalho, demonstrando as dificuldades operacionais para a adoção de uma lógica intersetorial. A identificação desses elementos e sua compreensão são passos importantes para a análise do porquê de algumas ações intersetoriais serem mais bem-sucedidas do que outras (Pires, 2016) (Quadro 1). 


\section{Modelo de gestão}

Os modelos de gestão e de organização das relações entre os setores interferem nas possibilidades de produção das ações intersetoriais. Eles definirão os níveis de gestão e de colaboração intersetorial nos ciclos de formulação, implementação e avaliação das políticas. Pode-se optar por maior grau de integração nos órgãos de cúpula (colaboração intersetorial na formulação), nas gestões locais (colaboração intersetorial na execução) ou em ambos os níveis (formulação e execução intersetoriais). Os modelos de gestão definirão "quem” (quais setores) participará das ações, "onde” (em qual território e para qual público) as ações serão desenvolvidas, "o que" (qual problema) será enfrentado. Definirão ainda o que será compartilhado - informações, responsabilidades, ações, orçamento - e em qual nível. Mesmo havendo um certo consenso em relação a algumas características fundamentais da colaboração intersetorial, as experiências analisadas pela literatura não apresentam um modelo de gestão único para a estratégia (Cunill Grau, 2014; Sposati, 2006). A diversidade de experiências e interpretações dos resultados relatados indicam que diferentes paradigmas e definições de sucesso orientam as ações, e que "não há uma verdade única" sobre o que constitui sucesso na colaboração intersetorial (Kuruvilla, et al., 2018). Há, no entanto, concordância de que é necessário um certo grau de inovação e de alteração das formas hegemônicas de organização do Estado. No Quadro 3, foi apresentada a experiência de programas de saúde em escolas australianas. Dois modelos de gestão coexistiram na colaboração intersetorial: o modelo centralizado do governo estadual da Austrália do Sul, Saúde em Todas as Políticas, que exigia aprovação de recomendações antes que as estratégias de todo o sistema fossem implementadas, em contraste com o modelo descentralizado da Educação.

Quadro 3 - Modelo de gestão, processo comunicativo e sustentabilidade em programas de saúde nas escolas australianas

Três estudos investigaram os mecanismos de integração e de colaboração intersetorial em programas de saúde desenvolvidos em escolas australianas (Burgess, et al., 2016; Eyk, et al., 2020; Tooher, et al., 2017).

Modelo de gestão: O governo estadual da Austrália do Sul implantou a estratégia Saúde em Todas as Políticas, com um modelo de gestão e de responsabilidades centralizado, exigindo a aprovação de recomendações antes que as estratégias de todo o sistema fossem implementadas. Em contraste, a Educação tinha um modelo descentralizado, com apoio para a implementação de políticas determinado em nível regional. Apesar das lógicas institucionais e modelos de gestão diferentes, os atores sustentaram as ações, seus produtos e resultados (Eyk, et al., 2020).

Processo comunicativo: Uma base sólida de comunicação e de relacionamentos profissionais interpessoais promoveu entendimentos compartilhados dos contextos sociais, organizacionais e políticos dos setores envolvidos. Foi fundamental para o alinhamento dos objetivos dos programas à missão dos setores e para a motivação dos participantes. As equipes colaborativas precisaram reservar um tempo para desenvolver esses entendimentos e para refletir sobre os respectivos pontos de vista (Tooher, et al., 2017).

Sustentabilidade: Dar visibilidade às ações e a seus resultados para todos os envolvidos foi uma forma de evidenciar a relevância e garantir a sustentabilidade dos programas. Apesar do sucesso, foram identificadas ameaças à continuidade de alguns programas (Burgess, et al., 2016). A inclusão de partes interessadas de todos os setores participantes, incluindo usuários, no planejamento e implementação mostrou-se estratégia garantidora da permanência das ações (Burgess, et al., 2016; Eyk, et al., 2020).

Fonte: Autores.

\section{Parceria}

Parcerias podem ser descritas como arranjos colaborativos nos quais atores de dois ou mais setores da sociedade (estado, mercado, sociedade civil) estão envolvidos em processos não hierarquizados, num esforço coletivo para a busca de objetivos comuns (Van Huijstee, et al., 2007). Esta é uma definição muito próxima do conceito de colaboração intersetorial utilizada neste texto. A parceria é, portanto, uma questão central na compreensão teórica e na prática intersetorial; é o instrumento fundamental por meio do qual serão gerados o apoio e a cooperação entre todos os envolvidos. Exige esforço dedicado, prontidão para colaborar e disponibilidade para atuar dentro de uma nova forma de organização. Algumas características fundamentais para o fortalecimento das parcerias são confiança entre as partes, boas relações de trabalho e presença de lideranças importantes. No entanto, na ação intersetorial ainda permaneceram como barreiras as diferentes prioridades setoriais e a falta de objetivos compartilhados no âmbito operacional (Holt, et al., 2018). 
De acordo com Russell e Smorodinskaya (2018), a forma mais eficaz de produzir inovação na atualidade envolve parcerias em rede. A contínua proliferação de redes em todo o mundo implica que bens, tecnologias e valores inovadores serão cada vez mais coproduzidos por atores/setores em rede que colaboram uns com os outros para formar sistemas relativamente sustentáveis de atores/setores, recursos e conexões (Russell \& Smorodinskaya, 2018) (Quadro 2).

\section{Processo comunicativo}

Processos comunicativos são os dispositivos de comunicação (verbal e não verbal) utilizados pelos indivíduos nos processos de interação. Compreendem a disponibilidade dos atores para o diálogo e para a utilização da argumentação, com reconhecimento das diferenças, respeito às falas, capacidade de aceitar e de discordar, apoio a argumentos válidos. Estas competências contribuem para se chegar ao entendimento mútuo e para a produção e coordenação das ações (Deslandes \& Mitre, 2009). A produção da colaboração intersetorial está embasada na capacidade dos indivíduos envolvidos construírem consensos em torno de um objetivo comum. Como prática inovadora, seu desenvolvimento depende da interação e do aprendizado desses indivíduos. Interação e aprendizagem serão favorecidas por meio de processos comunicativos com vistas à atualização de conhecimentos, técnicas e normas, em um contexto de relações simétricas, não coercitivo e com igualdade de possibilidades de falas (Rivera \& Artmann, 2016).

Como visto acima (Conflito), a explicitação e o enfrentamento das controvérsias fazem parte da construção da ação intersetorial. Isso só será possível por meio da interação mediada por processos comunicativos que possibilitarão: (i) estabelecimento de vínculos entre indivíduos e setores; (ii) geração de capacidade de negociação e de corresponsabilidade; (iii) fortalecimento da governança em rede; (iv) legitimação de processos e de porta-vozes; (v) delimitação de posições convergentes para a tomada de decisões e planejamento das ações (Bilodeau, et al., 2019) (Quadro 3).

\section{Setor}

Quando tratamos de políticas públicas, chamamos de setor cada uma das grandes áreas de atuação do aparato governamental: Assistência Social, Cultura, Educação, Obras, Saúde entre outros. A noção básica de colaboração intersetorial remete ao trabalho integrado de dois ou mais setores do governo para a solução de problemas sociais complexos (Bronzo, 2016; Inojosa, 2001).

A estrutura do Estado e a organização das instituições econômicas, com a divisão entre organizações públicas e privadas, dão origem a um segundo conceito de setor: setor público e setor privado empresarial e não empresarial (a sociedade civil). Neste aspecto, a colaboração intersetorial buscará a conciliação de interesses públicos e privados com a criação de novos formatos de governança que compõem as diferentes formas de parceria público-privada (Cunill Grau, 2014; Thamer \& Lazzarini, 2015).

Importante destacar que a colaboração intersetorial não substitui nem descarta a ação setorial com domínio técnico dentro de suas atribuições específicas. Pelo contrário; a atuação setorial com qualidade é fator agregador e gerador de confiança para os outros setores (Sposati, 2006). No entanto, se os formuladores de políticas olharem para fora das prioridades de seu campo setorial e perceberem como eles influenciam e são influenciados por outros, é provável que encontrem interesses comuns e alianças (inesperadas). Perceberão que a formulação de políticas mais integradas trará, muito provavelmente, resultados mais eficazes de desenvolvimento (Nilsson, et al., 2018).

\section{Sustentabilidade}

A sustentabilidade de uma política ou de um projeto se refere à continuidade de práticas eficazes no enfrentamento dos problemas elencados. Dessa forma, a sustentabilidade envolve o reconhecimento dos benefícios produzidos e a sua 
manutenção por meio da continuidade das atividades dentro de uma estrutura organizacional. O desafio fundamental é entender melhor como os esforços colaborativos podem ser adotados pelas burocracias formais e incorporados às estruturas e processos governamentais existentes (Bodin, 2017). Um outro componente da sustentabilidade é a construção de habilidades e de autonomia entre os receptores do programa (Shediac-Rizkallah \& Bone, 1998).

A sustentabilidade de projetos e políticas intersetoriais pressupõe o estabelecimento gradual de uma colaboração estável e duradoura, ao invés de uma relação vinculada a um projeto delimitado no tempo. Para que isso aconteça, múltiplos fatores inter-relacionados contribuirão para a continuidade do processo colaborativo: (i) os contextos político, organizacional e territorial nos quais as intervenções estão inseridas, privilegiando a descentralização com atuação nos espaços sociais, mais perto dos destinatários, com melhor diagnóstico dos problemas e, consequentemente, melhor planejamento de formas de enfrentamento; (ii) modelos inovadores de governança com compartilhamento de poder, de conhecimento, de responsabilidade e de orçamento; (iii) processo integrado de concepção e de implementação das intervenções; (iv) participação popular com envolvimento do público alvo, ao menos nas etapas de levantamento de problemas, planejamento e avaliação das ações; (v) o grau de transformação e o impacto sobre os problemas alvo (Cunill Grau, 2014; Hendriks, et al., 2015; Martins, et al., 2019; Shediac-Rizkallah \& Bone, 1998; Stirman, et al., 2012) (Quadro 3).

\section{Território}

Países, comunidades, indivíduos, todos se desenvolvem em um espaço. As sociedades são por natureza, entidades espaciais. Para além do aspecto físico, no entanto, o território deve ser entendido como a expressão e o produto das presenças, interações e relações entre os indivíduos. Territórios são, portanto, dinâmicos e polimorfos (Reis, 2015). Neste contexto, cada território terá sua situação peculiar, com suas potencialidades e fragilidades. Estas fragilidades, no mundo contemporâneo, não são poucas, e se expressam sob uma gama de riscos, vulnerabilidades e problemas sociais que se tornam os principais focos para as políticas públicas (Véras, 2010). Por estas questões, as políticas públicas devem ser, preferencialmente, formuladas para e implementadas nos territórios. Na medida em que, na sua origem e nas suas finalidades, as políticas estabelecem laços entre os atores locais e desenvolvem o seu capital social, elas criam condições para a estruturação, capacitação e qualificação do próprio território (Reis, 2015).

A literatura indica que a articulação intersetorial tem maior potência quando desenvolvida no âmbito do território e das famílias. Abordagens multidisciplinares integradas - baseadas no planejamento permanente, com ênfase territorial e participativa, envolvendo análise situacional, escolha de estratégias e tomada de decisões compartilhadas - poderão dar melhores respostas às questões dos territórios. Os múltiplos olhares contribuem para um trabalho em rede voltado para os problemas concretos, em oposição a um planejamento baseado exclusivamente em metas setoriais, elaboradas a partir de demandas ou necessidades genéricas (Nascimento \& Melazzo, 2013; Reis, 2015). A escolha fundamentada dos dados territoriais a serem utilizados é um fator de objetivação do trabalho intersetorial e de sustentabilidade para a iniciativa (Quadro 2).

\section{Dimensões da Colaboração Intersetorial}

As reflexões tecidas neste texto possibilitaram a análise da colaboração intersetorial sob a ótica de diferentes dimensões (Figura 1). A primeira delas é a dimensão política, relativa aos aspectos da tomada de decisões por trabalhar de maneira intersetorial na busca por soluções integradas para problemas complexos. A dimensão política é determinante do desenvolvimento técnico da colaboração intersetorial. Neste sentido a importância da agenda política e das formas adotadas de governança. Estes aspectos determinarão os modelos de gestão que orientarão a dimensão técnica da colaboração intersetorial. 
A dimensão técnica trata da operacionalização das ações intersetoriais, a partir dos modelos de gestão definidos pela dimensão política. O nível da ação é, inclusive, definido pelo modelo adotado. Aqui a importância dos operadores das políticas, das outras partes interessadas e dos contextos territoriais na produção de sinergias a partir das diferenças e das especificidades de cada indivíduo/setor.

A dimensão da interação - dimensão da vida social - modula as dimensões anteriores na perspectiva de viabilizar, com maior ou menor índice de sucesso, as redes de parcerias e os resultados planejados, por meio dos processos de comunicação e de interação. Em todas as três dimensões encontram-se mecanismos integradores, estimuladores e sustentadores da colaboração intersetorial.

Figura 1 - Modelo das dimensões da colaboração intersetorial a partir de seus conceitos estruturantes.

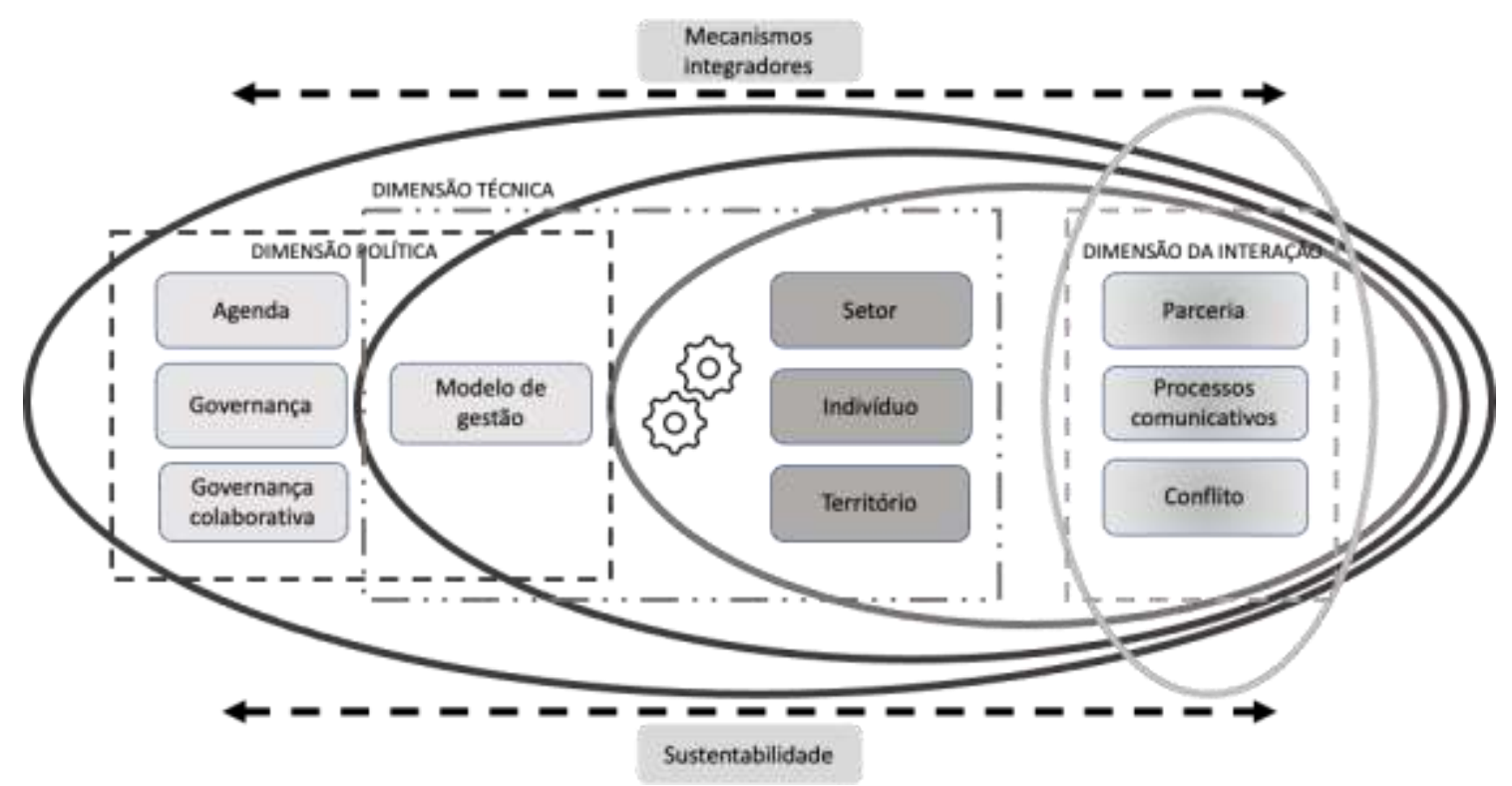

Fonte: Autores, a partir do modelo de capacidade de inovação das redes de negócios (Russell \& Smorodinskaya, 2018).

\section{Reflexões Sobre Contextos, Potencialidades e Práticas}

O glossário evidencia quão complexa pode ser a construção de uma colaboração intersetorial. Seja em sua dimensão política ou em sua dimensão técnica, os fatores envolvidos são muitos e se relacionam de forma dinâmica e intrincada. Trata-se de uma inovação que necessita de ferramentas de diferentes campos do conhecimento e cuja construção demanda uma rede de interações entre fatores contextuais e os múltiplos componentes da própria intervenção intersetorial. Neste sentido, maior envolvimento com pesquisadores de fora do setor saúde e o estabelecimento e fortalecimento de redes de evidências envolvendo formuladores de políticas, trabalhadores e a sociedade civil contribuirão sobremaneira para o entendimento do tema.

Se as políticas e os programas de saúde - e na opinião dos autores, também os sociais - funcionam como sistemas complexos (Gruen, et al., 2008), a colaboração intersetorial, enquanto possível estratégia para consecução dos objetivos destas políticas, também não é simples. Dessa forma, sua construção e sustentabilidade não serão viabilizadas sem conhecimento, esforço e tomada de decisões. Fomentar o debate em torno do tema fortalece as possibilidades de ações intersetoriais, aumentando a capacidade de resposta do Estado e das instituições às demandas sociais, à garantia de direitos e à consolidação da rede de proteção social. O modelo das dimensões da colaboração intersetorial que emergiu deste texto é relevante para todos os esforços colaborativos que buscam aperfeiçoar o trabalho conjunto e alcançar mudanças transformadoras positivas. 


\section{Agradecimentos}

Este estudo é parte de uma tese de doutorado desenvolvida na Faculdade de Odontologia da Universidade Federal de Minas Gerais. O projeto de pesquisa recebeu financiamento do Ministério da Saúde (TED MS/FNS nº 62/2012). O presente trabalho foi realizado com apoio da Coordenação de Aperfeiçoamento de Pessoal de Nível Superior - Brasil (CAPES) Código de Financiamento 001.

\section{Referências}

Akerman, M., Sá, R. F., Moysés, S., Rezende R. \& Rocha, D. (2014). Intersetorialidade? IntersetorialidadeS! Ciência \& Saúde Coletiva, $19(11), 4291-4300$. https://doi.org/10.1590/1413-812320141911.10692014.

Andrade, L. O. M. (2006). A saúde e o dilema da intersetorialidade. Hucitec.

Biblioteca Virtual em Saúde. Descritores em Ciências da Saúde. http://decs.bvs.br/cgi-bin/wxis1660.exe/decsserver/

Bilodeau, A., Galarneau, M., Lefebvre, C. \& Potvin, L. (2019). Linking process and effects of intersectoral action on local neighbourhoods: systemic modelling based on Actor-Network Theory. Sociology of Health \& Illness, 41(1), 165-179. https://doi.org/10.1111/1467-9566.12813.

Bodin, Ö. (2017). Collaborative environmental governance: achieving collective action in social-ecological systems. Science, 357(6352), 659-668. https://doi.org/10.1126/science.aan1114.

Brasil. (2014). Política Nacional de Promoção da Saúde. Revisão da Portaria MS/GM n. 687, de 30 de março de 2006. Ministério da Saúde.

Brasil, E. G. M., Silva, R. M., Silva, M. R. F., Rodrigues, D. P. \& Queiroz, M. V. O. (2017). Promoção da saúde de adolescentes e Programa Saúde na Escola: complexidade na articulação saúde e educação. Revista da Escola de Enfermagem da USP, 51:e03276. https://doi.org/10.1590/S1980-220X2016039303276.

Bronzo, C. (2016). Sistema de proteção social integral com foco em direitos e suas implicações para a integração entre benefícios e serviços na proteção social não contributiva no Brasil: uma reflexão exploratória. In Macedo, J. M., Xerez, F. H. S., Lofrano, R. (Org.), Cadernos de estudo. Desenvolvimento social em debate. Intersetorialidade nas políticas sociais: perspectivas a partir do Programa Bolsa Família (pp. 103-126). Imprensa Nacional.

Burgess, T., Braunack-Mayer, A., Tooher, R., Collins, J., O’Keefe, M., Skinner, R. et al. (2016). Optimizing intersectoral collaboration between health and education: the Health Bridges study. Journal of Public Health, 38(4), 430-7. https://doi.org/10.1093/pubmed/fdv190.

Canadian Council on Social Determinants of Health. (2014). Maps to inform intersectoral planning and action. Technical report.

Chiari, A. P. G., Ferreira, R. C., Akerman, M., Amaral, J. H. L., Machado, K. M. \& Senna, M. I. B. (2018). Rede intersetorial do Programa Saúde na Escola: sujeitos, percepções e práticas. Cadernos de Saúde Pública, 34(5), 1-15. https://doi.org/10.1590/0102-311X00104217.

Chiari, A. P. G., Senna, M. I. B., Soares, A. R. S., Ferreira, R. C., Alves, C. R. L., Rubió, F. P. \& Cury, G. C. (2021). Desenvolvimento infantil integral e integrado: análise preliminar de uma ação educativa intersetorial. In Anais do $4^{\circ}$ Congresso Brasileiro de Política, Planejamento e Gestão da Saúde 2021, Rio de Janeiro. Galoá. https://proceedings.science/cbppgs-2021/papers/desenvolvimento-infantil-integral-e-integrado--analise-preliminar-de-uma-acaoeducativa-intersetorial?lang=pt-br.

Chircop, A., Bassett, R. \& Taylor, E. (2015). Evidence on how to practice intersectoral collaboration for health equity: a scoping review. Critical Public Health, 25(2), 178-91. https://doi.org/10.1080/09581596.2014.887831

Cunill Grau, N. (2005). La intersectorialidad en el gobierno y gestión de la política social. X Congreso Internacional del CLAD sobre la Reforma del Estado y de la Administración Pública, Santiago, Chile.

Cunill Grau, N. (2014). La intersectorialidad en las nuevas políticas sociales. Un acercamiento analítico-conceptual. Gestión y Política Pública, 23(1), 5-46.

Deslandes, S. F. \& Mitre, R. M. (2009). A. Communicative process and humanization in healthcare. Interface - Comunicação, Saúde, Educação, 13(1), 64164. https://doi.org/10.1590/S1414-32832009000500015.

Dubois, A., St-Pierre, L. \& Veras, M. (2015). A scoping review of definitions and frameworks of intersectoral action. Ciência \& Saúde Coletiva, 20(10), 29332942. https://doi.org/10.1590/1413-812320152010.01222014.

Eyk, H., Delany-Crowe, T., Lawless, A., Baum, F., MacDougall, C. \& Wildgoose, D. (2020). Improving child literacy using South Australia's Health in All Policies approach. Health promotion International, 35(5), 958-72. https://doi.org/10.1093/hepro/daz013.

Ferreira, I. R. C., Moysés, S. J., França, B. H. S., Carvalho, M. L. \& Moysés, S. T. (2014). Percepções de gestores locais sobre a intersetorialidade no Programa Saúde na Escola. Revista Brasileira de Educação, 19(56), 61-76. https://doi.org/10.1590/S1413-24782014000100004.

Freiler, A., Muntaner, C., Shankardass, K., Mah, C. L., Molnar, A., Renahy, E. \& O’Campo, P. (2013). Glossary for the implementation of Health in All Policies (HiAP). Journal of Epidemiology and Community Health, 67, 1068-1072. https://doi.org/10.1136/jech-2013-202731.

Glandon, D., Mondal, S., Okeyo, I., Zaidi, S., Khan, M. S., Dar, O. \& Bennett, S. (2019). Methodological gaps and opportunities for studying multi.sectoral collaboration for health in low- and middle-income countries. Health Policy and Planning 34(2), 7-17. https://doi.org/10.1093/heapol/czz116.

Gruen, R. L., Elliott, J. H., Nolan, M. L., Lawton, P. D., Parkhill, A., McLaren, C. J. \& Lavis, J. N. (2008). Sustainability science: an integrated approach for health-programme planning. Lancet, 372, 1579-1589. https://doi.org/10.1016/S0140-6736(08)61659-1. 
Guba, E. G. \& Lincoln, Y. S. (2011). Avaliação de quarta geração. Editora da Unicamp.

Hendriks, A. M., Jansen, M. W. J., Gubbels, J. S., De Vries, N. K. Molleman, G. \& Kremers, S. P. J. (2015). Local government officials' views on intersectoral collaboration within their organization - A qualitative exploration. Health Policy and Technology, 4, 47-57. https://doi.org/10.1016/j.hlpt.2014.10.013.

Holt, D. H., Rod, M. H., Waldorff, S. B. \& Tjørnhøj-Thomsen, T. (2018). Elusive implementation: an ethnographic study of intersectoral policymaking for health. BMC Health Services Research, 18(54), 1-12. https://doi.org/10.1186/s12913-018-2864-9.

Inojosa, R. M. (2001). Sinergia em Políticas e Serviços Públicos: desenvolvimento social com intersetorialidade. Cadernos Fundap, 22, 102-110.

Kuruvilla, S., Hinton, R., Boerma, T., Bunney, R., Casamitjana, N., Cortez, R. et al. (2018). Business not as usual: how multisectoral collaboration can promote transformative change for health and sustainable development. BMJ, 363:k4771. https://doi.org/10.1136/bmj.k4771.

Latour, B. (2012). Reagregando o social: uma introdução à teoria do Ator-Rede. Salvador, BA: EDUFBA.

Martins, H. F., Mota, J. P. \& Marini, C. (2019). Modelos de negócio na esfera pública: o modelo canvas de governança pública. Cadernos EBAPE.BR, 17(1), 49-67. https://doi.org/10.1590/1679-395167893.

Martuccelli, D. (2002). Grammaires de l'individu. Gallimard.

McDaid, D. \& Park, A. (2016). Evidence on financing and budgeting mechanisms to support intersectoral actions between health, education, social welfare and labour sectors. Copenhagen, Dinamarca: WHO Regional Office for Europe.

Monnerat, G. L. \& Souza, R. G. (2011). Da Seguridade Social à intersetorialidade: reflexões sobre a integração das políticas sociais no Brasil. Revista Katálysis, 14(1), 41-49. https://doi.org/10.1590/S1414-49802011000100005.

Monnerat, G. L. \& Souza, R. G. (2014). Intersetorialidade e Políticas Públicas: um diálogo com a literatura atual. In G. L. Monnerat, N. L. T. Almeida, \& R. G. Souza (Org.), A intersetorialidade na agenda das Politicas Sociais (pp. 41-54). Papel Social.

Nascimento, P. F. \& Melazzo, E. S. (2013). Território: conceito estratégico na assistência social. Serv. Soc. Rev., 16(1), 66-88. https://doi.org/10.5433/16794842.2013v16n1p66.

Nilsson, M., Chisholm, E., Griggs, D., Howden-Chapman, P., McCollum, D., Messerli, P., Neumann, B., Stevance, A. S., Visbeck, M. \& Stafford-Smith, M. (2018). Mapping interactions between the sustainable development goals: lessons learned and ways forward. Sustainability Science, 13, 1489-1503. https://doi.org/10.1007/s11625-018-0604-z.

Oliveira, A. G. \& Pisa, B. J. (2015). IGovP: índice de avaliação da governança pública - instrumento de planejamento do Estado e de controle social pelo cidadão. Revista de Administração Pública, 49(5), 1263-1290. https://doi.org/10.1590/0034-7612136179.

Pires, R. R. C. (2016). Intersetorialidade, arranjos institucionais e instrumentos da ação pública. In Macedo, J. M., Xerez, F. H. S., Lofrano, R. (Org.), Cadernos de estudo. Desenvolvimento social em debate. Intersetorialidade nas políticas sociais: perspectivas a partir do Programa Bolsa Família (pp. 67-80). Brasília, DF: Imprensa Nacional.

Ran, B. \& Qi, H. (2018). Contingencies of power sharing in collaborative governance. American Review of Public Administration, 48(8), 836-851. https://doi.org/10.1177/0275074017745355.

Rasanathan, K., Bennett, S., Atkins, V., Beschel, R., Carrasquilla, G., Charles, J. et al. (2017). Governing multisectoral action for health in low- and middleincome countries. PLoS Med 14(4): e1002285. https://doi.org/10.1371/journal. pmed.1002285.

Reis, J. (2015). Território e políticas do território. A interpretação e a ação. Finisterra, 100, 107-122. httos://doi.org/10.18055/Finis7868.

Rivera, F. J. U. \& Artmann, E. (2016). Inovação a agir comunicativo: redes e tecnologias de gestão para a saúde. Cadernos de Saúde Pública, 32(2), 1-11. https://doi.org/10.1590/0102-311X00177014.

Russell, M. G. \& Smorodinskaya, N. V. (2018). Leveraging complexity for ecosystemic innovation. Technological Forecasting \& Social Change, 136, 114131. https://doi.org/10.1016/j.techfore.2017.11.024.

Santos, M. H. C. (1997). Governabilidade, governança e democracia: criação de capacidade governativa e relações executivo-legislativo no Brasil pósconstituinte. Dados, 40(3). https://doi.org/10.1590/S0011-52581997000300003.

Shankardass, K., Solar, O., Murphy, K., Greaves, L. \& O’Campo, P. (2012). A scoping review of intersectoral action for health equity involving governments. International Journal of Public Health, 57(1), 25-33. https://doi.org/10.1007/s00038-011-0302-4.

Shediac-Rizkallah, M. C. \& Bone, L. R. (1998). Planning for the sustainability of community-based health programs: conceptual frameworks and future directions for research, practice and policy. Health Education Research, 13(1), 87-108. https://doi.org/10.1093/her/13.1.87.

Simmel, G. (1995). Le conflit. Dijon-Quetigny, Circé.

Solar, O., Valentine, N., Albrech, D. \& Rice, M. (2009). Moving forward to Equity in Health: what kind of intersectoral action is needed? An approach to an intersectoral typology. 7th Global Conference for Health Promotion, Nairobi, Kenya.

Sousa, M. C., Esperidião, M. A. \& Medina, M. G. (2017). A intersetorialidade no Programa Saúde na Escola: avaliação do processo político-gerencial e das práticas de trabalho. Ciência \& Saúde Coletiva, 22(6), 1781-90. https://doi.org/10.1590/1413-81232017226.24262016.

Sposati, A. (2006). Gestão pública intersetorial: sim ou não? Comentários de experiência. Serviço Social \& Sociedade, 85, $133-141$. 
Research, Society and Development, v. 10, n. 9, e28610918054, 2021

(CC BY 4.0) | ISSN 2525-3409 | DOI: http://dx.doi.org/10.33448/rsd-v10i9.18054

Stirman, S. W., Kimberly, J., Cook, N., Calloway, A., Castro, F. \& Charns, M. (2012). The sustainability of new programs and innovations: a review of the empirical literature and recommendations for future research. Implementation Science, 7(17), 1-19. https://doi.org/10.1186/1748-5908-7-17.

Thamer, R. \& Lazzarini, S. G. (2015). Projetos de parceria público-privada: fatores que influenciam o avanço dessas iniciativas. Revista de Administração Pública, 49(4), 819-846. https://doi.org/10.1590/0034-7612119746.

Tooher, R., Collins, J., Braunack-Mayer, A., Burgess, T., Skinner, S. R., O’Keefe, M., Watson, M. \& Marshall H. S. (2017). Intersectoral collaboration to implement school-based health programmes: Australian perspectives. Health Promotion International, 32, 312-21. https://doi.org/10.1093/heapro/dav120.

United Nations. (2019). Sustainable development goal 17: strengthen the means of implementation and revitalize the global partnership for sustainable development [online]. New York, Estados Unidos da América. https://sustainabledevelopment.un.org/sdg17

Van Huijstee, M. M., Francken, M. \& Leroy, P. (2007). Partnerships for sustainable development: a review of current literature. Environmental Sciences, 4(2), 75-89. https://doi.org/10.1080/15693430701526336.

Véras, M. P. B. (2010). Cidade, vulnerabilidade e território. Ponto-e-Vírgula, 7, 32-48. https://doi.org/10.23925/1982-4807.2010i7p\%p. 